\title{
Psico-oncologia: A atuação do Psicólogo junto aos familiares e ao paciente oncológico
}

\section{Psycho-oncology: The Psychologist's work with family members and the cancer patient}

\author{
Eleni Severino dos Santos Pio ${ }^{\dagger}$, Maria Clara de Mello Andrade*
}

Como citar esse artigo. PIO, E.S.S; ANDRADE, M.C.M. Psico-oncologia: A atuação do Psicólogo junto aos familiares e ao paciente oncológico. Revista Mosaico, v.11, n.1, p. 93-99, 2020 .

\begin{abstract}
Resumo
O câncer é uma patologia que pode interferir de forma significativa na vida do sujeito e de seu familiar/cuidador. Neste contexto, surge a Psico-oncologia, uma área de interface entre a Psicologia e a Oncologia. O presente estudo busca analisar o papel da Psico-oncologia na trajetória do doente e de sua família no processo de adoecimento do câncer. Para tanto, foi realizada uma pesquisa bibliográfica, através da busca ativa de informações em livros, revistas e artigos acadêmicos. A partir do levantamento da literatura revisada, destacamos que a atuação do psicólogo no cuidado oncológico se dá por meio do apoio psicossocial e psicoterapêutico através de grupos de apoios, acolhimento, assistência e escuta, entre outras práticas, visando propiciar um melhor enfrentamento da doença entre os envolvidos. De modo geral, pode-se inferir que a Psico-oncologia é uma ferramenta contemporânea e indispensável na promoção da qualidade de vida ao paciente com câncer, a sua família e à equipe que o acompanha.
\end{abstract}

Palavras-chave: Câncer, Família, Paciente, Psico-oncologia.

\begin{abstract}
Cancer is a condition that can significantly interfere with the life of the subject and his / her family member / caregiver. In this context comes the psycho-oncology, an interface area between psychology and oncology. This study aims to analyze the role of psycho-oncology in the trajectory of patients and their families in the process of cancer illness. To this end, a bibliographic search was performed through the active search for information in books, magazines and academic articles. From the survey of the reviewed literature, we highlight that the role of the psychologist in cancer care, is through psychosocial and psychotherapeutic support through support groups, welcoming, assistance, listening, among other practices, aiming to provide a better coping with the disease among those involved. In general, it can be inferred that psycho-oncology is a contemporary and indispensable tool in promoting the quality of life for cancer patients, their families and their accompanying staff.

Keywords: Cancer. Family, Patient, Psycho-oncology.
\end{abstract}

\section{Introdução}

O câncer é reconhecido como uma doença crônico-degenerativa que atinge milhões de pessoas no mundo. É uma patologia que pode interferir de forma significativa na vida do sujeito e de seu familiar/ cuidador. Neste contexto, surge a Psico-oncologia, uma área de interface entre a Psicologia e a Oncologia. (INCA, 2014)

É perceptivel, no decorrer dos anos, que o diagnóstico do câncer tem causado desequilíbrio psicológico, tanto nas pessoas que o portam, quanto em seus familiares. Ao receber um diagnóstico de uma doença como o câncer a família poderá ser abalada levando todos os seus membros a vivenciarem de forma singular esse fato. Tal vivência carrega interrupções.
Faz com que fenômenos que antes não haviam sido experienciados ou enfrentados surjam de forma imperativa. (NEME, 2010). Diante do exposto, surge o seguinte questionamento: como se dáo trabalho dos psicólogos frente à problemática do câncer e todo o processo de adoecimento vivenciado pelo paciente e pela sua família?

A fim de responder tal questionamento este artigo de revisão bibliográfica especializada, tem como objetivo analisar o papel da Psico-oncologia na trajetória do doente e da família no processo de adoecimento, visando identificar as dificuldades que estes vêm enfrentando ao receber o diagnóstico, nas diferentes fases de tratamento e evolução do câncer. Os objetivos específicos são: contextualizar a família e o câncer; descrever a atuação do psicólogo oncológico e as contribuições ofertadas aos pacientes, familiares e a

\footnotetext{
Afiliação dos autores:

${ }^{\dagger}$ Psicóloga, Vassouras, RJ, Brasil

* Professora Asssistente II do Curso de Psicologia da Universidade de Vassouras, Vassouras, RJ, Brasil
}

*Email de correspondência: mariamelloandrade@hotmail.com 
equipe multidisciplinar.

Para tanto, foi realizada uma pesquisa bibliográfica, através da busca ativa de informações em livros, revistas e artigos acadêmicos, todos da área da Psicologia. Abordamos alguns aspectos gerais referentes ao câncer, as reações que ocorrem perante o diagnóstico e tratamento, bem como o impacto que esta doença causa sobre a vida do sujeito e do sistema familiar. Falamos sobre o desenvolvimento da Psicooncologia, entendida como uma área específica da psicologia da saúde, voltada para o estudo do câncer e trazemos algumas considerações sobre a importância e as possibilidades de atuação em vários aspectos, como: prevenção do câncer, paciente oncológico, família e equipe multidisciplinar.

Este trabalho justifica-se devido ao câncer ser uma patologia grave e de grande acometimento psicossocial, sendo a Psico-oncologia um tema atual e em formação no Brasil. Neste contexto, é necessário que o psicólogo que atua no cuidado oncológico compreenda o doente e a família, seus comportamentos e sentimentos a fim de intervir de forma adequada.

Este estudo poderá contribuir primordialmente ao processo de enfrentamento do câncer, considerando a necessidade do acompanhamento psicológico e a possibilidade de auxílio para melhor enfrentamento e qualidade de vida do doente e de seus familiares.

\section{Conhecendo o Câncer}

Segundo a Organização Mundial da Saúde (OMS), o câncer é considerado uma doença crônica degenerativa das mais temidas evem se contextualizando como um importante problema de saúde pública em países desenvolvidos e em desenvolvimento (BRASIL, 1977).

O Instituto Nacional de Câncer (INCA) conceitua o câncer como um conjunto de mais de cem doenças que têm em comum o crescimento desordenado de células que invadem os tecidos e órgãos, podendo espalhar-se para outras regiões do corpo, num processo denominado metástase. Dividindo-se rapidamente, estas células tendem a ser muito agressivas e incontroláveis, determinando a formação de tumores, definidos como acúmulo de células cancerosas ou neoplasias malignas. Por outro lado, um tumor benigno significa simplesmente uma massa localizada de células que se multiplicam vagarosamente e se assemelham ao seu tecido original, raramente constituindo um risco de vida. (INCA, 2014)

As causas do câncer estão relacionadas a um conjunto de fatores de natureza intrínseca e extrínseca. Entre os fatores intrínsecos estão a idade, o sexo, a etnia ou raça, e a herança genética. Já no grupo de fatores extrínsecos estão incluídos o uso de tabaco e álcool, hábitos alimentares inadequados, inatividade física, imunossupressão, exposições ocupacionais, obesidade, uso de drogas, fatores ambientais, reprodutivos, ocupacionais e hormonais. (PEÇANHA, 2005).

É pertinente ressaltar que o câncer é uma doença que provoca impacto psicológico, pois muitas vezes pode representar uma caminhada dolorosa e progressiva para a mutilação e a morte. Hoje, segundo dados do INCA, o câncer representa uma das principais causas de morte no mundo. Estima-se que em 2030 a ocorrência de 21,4 milhões de casos novos de câncer e 13,2 milhões de mortes por câncer, em consequência do crescimento e desenvolvimento da população. (INCA, 2014)

Complementando, segundo Kovacs (1992) o câncer também é chamado de "gravidez demoníaca" por causa do seu crescimento desorganizado. $\mathrm{O}$ tratamento é brutal e muitas vezes pior que a doença. Desta forma, são usadas metáforas de guerra para nomear estes tratamentos como: guerra química para quimioterapia, e guerra de mísseis para a radioterapia.

Opaciente comcâncerem processo de tratamento passa a sofrer perdas em sua qualidade de vida pelos sintomas da doença, mas também em consequência dos efeitos adversos do tratamento quimioterápico e radioterápico. Dor, exames invasivos, alterações na imagem corporal, mudanças de papel social, isolamento e dependência de cuidadores, além das incertezas associadas ao tratamento, aumentam a vulnerabilidade de pacientes e familiares para transtornos mentais. (SAWADA et al., 2009)

Devido às formas de tratamento, o câncer leva, na maioria das vezes, a necessidade de internação e intervenção cirúrgica. Porém, não se deve esquecer que a hospitalização pode ser uma vivência complicada para qualquer ser humano, pois gera angústia por estar em um ambiente estressante, onde até receber apoio é limitado. A presença do familiar/acompanhante é indispensável, entretanto envolve mudanças na rotina de toda a família (FAQUINELLO et al., 2007).

Segundo Froelich (2011) independente da faixa etária e/ou tipo, o câncer carrega consigo a iminência da ideia de fatalidade e questões ligadas com mudanças físicas e imagem corporal, entre outras crenças influentes no tratamento, onde a ansiedade, o medo e as fantasias sobre a doença terão de ser enfrentadas.

\section{A família e o paciente diante do câncer}

A doença crônica tem um impacto sobre a vida do sujeito e do seu familiar/ cuidador. Neste contexto, podemos inferir que a família é um "agente de extensão" no tratamento oncológico, e também um alicerce de suporte psicossocial. O acolhimento da família é muito importante, para que o paciente se sinta apoiado. É essa relação familiar que irá contribuir, especialmente, para 
o enfrentamento de uma doença grave como o câncer, mudando a vida do sujeito de forma singular e também todo ambiente no qual este está inserido. (DIAS; DURÁ, 2001)

Segundo Kluber-Ross (2005) no período da doença, os familiares desempenham papel preponderante, e suas reações muito contribuem para a própria reação do paciente. Logo os sentimentos de perda e de insegurança e a instabilidade emocional tomarão conta da vida deste familiar/cuidador, que passa a ter atribuições que outrora eram compartilhadas com o sujeito adoentado.

Fases, como as citadas por Kluber-Ross (2005), serão vivenciadas de forma subjetiva, tanto pelo indivíduo adoentado quanto por seu cuidador/familiar. Estes poderão recusar o diagnóstico, entrar em desespero e até mesmo se aceitar nesta condição de doente ou de familiar/cuidador, pois ter ou ser um doente na família é fugir do padrão da expectativa de que tudo é perfeito. Ambos poderão vivenciar momentos de negação, raiva, barganha, depressão e aceitação, e poderão se questionar: porque comigo? Na minha família? Adaptar-se e aceitar esta condição pode levar tempo e depende sempre de como estes atores irão encarar cada fato.

Não existe uma sequência a ser seguida ao vivenciar estes estágios, pois cada sujeito vive as fases de forma subjetiva, tanto o paciente quanto a família. É necessário analisar quem é este paciente que se encontra adoentado, qual o papel dele no círculo familiar e em qual contexto social ele está inserido. Todas estas questões são de suma importância para se saber qual será o impacto do câncer sobre este sujeito e qual serão as reais vivências do adoecimento. (KLUBER-ROSS, 2005) Diante do câncer a família poderá apresentar sua própria dinâmica, ou seja, seu funcionamento alterado e seu espaço reestruturado, visando a reelaboração de sonhos e planos (COSTA, 2012).

Complementando, a existência da doença na família pode mobilizar sentimentos positivos e negativos que precisam ser trabalhados por todos. O câncer pode abalar emocionalmente e fisicamente o contexto familiar, uma vez que há mudança na rotina de todos os envolvidos. É importante ressaltar que o abalo familiar dependerá muito de quem é este membro adoentado, não que um tenha mais importância que outro, mas o pesar será manifesto de forma diferente se o doente for uma criança ou um provedor daquele ambiente, por exemplo. (MELMAN, 2001)

Peçanha (2005) indaga que as doenças crônicas, como o câncer, podem levar o indivíduo e sua família a deparar-se com a possibilidade de morte, o que provoca intensas alterações na rotina. No domicílio, o paciente oncológico muitas vezes apresenta um sentimento de ser um peso para a sua família, e esta, por não saber como enfrentar a doença ou mesmo devido ao excesso de zelo, considera o paciente incapaz para decidir sobre sua vida e sua doençaou, até mesmo, de encarar a gravidade da doença.

Na visão de Froelich(2011) o surgimento de um câncer, para o doente e sua família, pode ser considerado como um divisor da existência: há sempre um antes e um depois da doença, tanto para os sucessos como para os fracassos, tanto para as transformações positivas que possam ocorrer como para as negativas. Neste sentido, mesmo o câncer sendo uma patologia envolta por muitos mitos e verdades, vale ressaltar que muitos casos têm cura e se descobertos a tempo estes são tratáveis. A dedicação e o empenho do doente e de sua família podem transformar o câncer em um ponto de transformação nas suas vidas. Assim, o adoecer de câncer vai além de um diagnóstico sombrio, de uma perspectiva de morte; ele poderá ser visto e enfrentado como um grande desafio para o doente e sua família.

\section{A Psico-oncologia}

Costa (2001) argumenta que no decorrer dos últimos anos a psico-oncologia se constituiu como uma importante e indispensável ferramenta para a promoção das condições de qualidade de vida do paciente com câncer, facilitando o processo de enfrentamento de eventos estressantes, relacionados ao processo de tratamento da doença. Desta forma:

Ao contrário do que ocorria no passado, quando indivíduos com diagnóstico de câncer eram estudados apenas em termos de quantos sobreviviam, e por quanto tempo, [...] hoje a interface Psicologia-Oncologia traz à tona uma preocupação mais ampla: a qualidade de vida da pessoa com câncer (GIMENES, 2003, p. 35).

Veit e Carvalho (2010) relatam que a Psicooncologiase originou nos Estados Unidos, em um grande centro médico especializado em câncer, o Memorial Sloan Kettering Hospital, de New York e que nasceu da constatação de que fatores psicológicos e comportamentais estão envolvidos na etiologia do câncer e no seu desenvolvimento.O termo psicooncologia é formado por: psico (mente), onco (tumor) e logia (conhecimento, estudo). O conhecimento sobre a Psico-oncologia foi introduzido no Brasil através de palestras de Maria Margarida M.J. de Carvalho, em 1984. Ainda, segundo o autor, o I Encontro Brasileiro de Psico-Oncologia ocorreu em 1989 em Curitiba, e contribuiu para o desenvolvimento da área. (VEIT; CARVALHO, 2010)

Neme (2010) afirma que a psico-oncologia representa uma ampla área de estudos e de atuação profissional e tem como principais objetivos:

1) A prevenção do câncer, o tratamento e a assistência integral ao paciente oncológico e seus familiares; 
2) A formação de profissionais de saúde;

3) A realização de pesquisas que possibilitem a sistematização dos conhecimentos produzidos e que conduzam a novos caminhos.

Nesta perspectiva, Gimenes (2003) define a psico-oncologia como uma área de interface entre a oncologia e a psicologia, tomando por base concepções de saúde e doença inerentes ao modelo biopsicossocial. Complementando este conceito, Costa (2001) descreve a psico-oncologia como um campo interdisciplinar da saúde que estuda a influência de fatores psicológicos sobre o desenvolvimento, o tratamento e a reabilitação de pacientes com câncer.

Em síntese, Gimenes (2003, p.17) relata que a psico-oncologia é uma área que "compreende todas as intervenções psicológicas possíveis no trabalho com o paciente com câncer e seus familiares".

A psico-oncologia reconhece que o aparecimento e a evolução de doenças como o câncer necessitam de um nível de compreensão e ação que transcendam o modelo biomédico-mecanicista tradicional, buscando esclarecer os fatores biológicos e sociais implicados na gênese e nos tratamentos do câncer, bem como elucidando os aspectos psicológicos envolvidos na doença, em sua remissão ou recidiva e em seu impacto nas famílias e nos profissionais de saúde. (NEME, 2010, p. 20).

Neste sentido, a psico-oncologia tornou-se uma importante ferramenta de promoção à saúde e avaliação dos processos psicológicos envolvidos no surgimento e desenvolvimento do câncer, e também na efetivação da qualidade de vida, na avaliação e identificação do papel psicossocial da prevenção e do tratamento do câncer.

\section{Atuação do Psicólogo no Tratamento Oncológico}

A psicologia é uma das profissões da saúde cuja inclusão em equipes de acompanhamento de pacientes com câncer é regulamentada por lei, através da Portaria $\mathrm{n}^{\mathrm{o}} 3.535$ do Ministério da Saúde, publicada em 14 de outubro de 1998, que determina que toda equipe responsável pelo tratamento de pessoas com câncer tenha, entre seus profissionais, um psicólogo. (SAMPAIO; LOHR, 2008).

A atuação do psicólogo no ambiente hospitalar é reconhecida pelo Conselho Federal de Psicologia (CFP) através da Resolução $n^{\circ}$ 013/2007, a qual especifica algumas de suas atribuições:

[...] Atende a pacientes, familiares e/ou responsáveis pelo paciente; membros da comunidade dentro de sua área de atuação; membros da equipe multiprofissional e eventualmente administrativa, visando o bem estar físico e emocional do paciente; [...] tendo como sua principal tarefa à avaliação e acompanhamento de intercorrências psíquicas dos pacientes que estão ou serão submetidos a procedimentos médicos, visando basicamente àpromoção e/ou a recuperação da saúde física e mental. Promove intervenções direcionadas à relaçãomédico/paciente, paciente/família, e paciente/paciente e do paciente em relação ao processo do adoecer, hospitalização e repercussões emocionais que emergem neste processo. (CFP, 2007, p. 21-22)

Nesta perspectiva, Neme(2010) enfatiza que equipes formadas por psicólogos começaram a ser requisitadas pelos oncologistas, inicialmente com o objetivo de auxiliar o médico na dificuldade da informação do diagnóstico de câncer ao paciente e a sua família. Desta forma, Campos diz que:

O estigma e os mitos que envolviam o câncer puderam
ser substituídos por conhecimento e instrumentos
deenfrentamento tais como os tratamentos, grupos de
autoajuda, e suporte psicossocial. A partir desses fatos foi
possívelexplorar e estudar respostas e comportamentos
psicológicos associados ao câncer. (CAMPOS, 2010, p.61)

De acordo com Simonetti (2004), é preciso descobrir a verdade do paciente sobre a doença. Nesta visão é fundamental que "o psicólogo trabalhe com o sentido das coisas, não com a verdade das coisas" (INCA, 2014, p. 41), elaborando estratégias precisas de um tratamento psicológico, escutando o que se passa com o paciente, as suas histórias, expectativas, medos e experiências.

Costa (2001) evidencia que o psicólogo deve priorizar a promoção de mudanças de comportamento relacionadas à saúde do indivíduo e de seus familiares. Complementando, o autor relata que a experiência de tratamento deve se constituir em uma condição de aprendizagem sociocomportamental e cognitiva para o paciente.

Cabe ao psicólogo demonstrar que os repertórios decomportamentos adquiridos no contexto do tratamento podem serúteis em diversas situações de risco, mesmo aquelas distantes do contexto de doenças e tratamentos médicos, a que o indivíduo for submetido. (COSTA, 2001, p.37)

Desta forma, o papel do psicólogo no âmbito oncológico é o de proporcionar apoio psicossocial e psicoterapêutico diante do processo de adoecimento do câncer, demostrando possíveis estratégias de auxílio para um melhor enfrentamento, qualidade de vida e maiores expectativas de sobrevivência para os pacientes e seus familiares. Assim, a psico-oncologia fundamenta-se em uma atuação preventiva e humanizada com o intuito de buscar formas de tratamento dignas, favorecendo o processo de tratamento.

Em seus estudos, Costa (2001) explicita que a atuação do psicólogo oncológicodeve ser pautada tanto no apoio, no aconselhamento e na reabilitação, quanto na psicoterapia individual/grupal, facilitando a transmissão do diagnóstico e a melhor aceitação dos tratamentos e 
proporcionando melhor qualidade de vida e, no caso de paciente em fase terminal, uma melhor maneira de morrer. Nesta mesma linha, Gimenes (2003) afirma que as intervenções psicológicas são imprescindíveis, pois têm a possibilidade de ajudar o paciente a lidar com os aspectos que abrangem o diagnóstico de uma doença que está relacionada ao sofrimento, ao medo da morte, a mutilação e a solidão. Liberato e Carvalho (2008), indagam que a psico-oncologiaaborda:

[...] impacto do câncer nas funções psicológicas do paciente, da família e da equipe de saúde (unidade cuidadora), e o papel que as variáveis psicológicas e comportamentais têm no risco do câncer e na sobrevivência dos pacientes oncológicos. Além de dar espaço para que o paciente traga o que quiser trazer. (LIBERATO; CARVALHO, 2008, p. 342)

Sampaio e Lohr (2008) afirmam que no tratamento de pacientes com diagnóstico de câncer o psicólogo se empenha por minimizar os efeitos causados pela doença, de modo a facilitar a reintegração desse paciente à sociedade e a uma rotina mais próxima possível da que tinha antes do diagnóstico. Desse modo, evita-se o surgimento de complicações de ordem psicológica que possam interferir no campo profissional, afetivo e social, tanto do sujeito em tratamento quanto na de seus familiares.

De acordo com a abordagem de Veit e Carvalho (2010), os temas que mais preocupam os pacientes com câncer e que devem ser focos das intervenções psicoterápicas são: sensação de falta de controle sobre a própria existência, temor da solidão e da própria morte, sentimento de impotência e fracasso e temor dos efeitos adversos do tratamento oncológico.

Neste contexto, Sampaio e Lohr (2008) apontam que a empatia e a escuta acolhedora verbal e nãoverbal devem estar presentes no contexto do psicólogo oncológico, permitindo que o paciente possa se confrontar com seus conteúdos internos, suas angústias e sentimentos em geral, para que, a partir daí, inicie o processo de aceitação, elaboração e superação no que diz respeito ao adoecimento, permitindo ao psicólogo identificar as reais demandas do paciente. Desta forma, a escuta do sujeito "pode ser então uma importante ferramenta para a ressignificação do seu sofrimento e um caminho para o estabelecimento de novos recursos psíquicos de enfrentamento ou de fortalecimento" (INCA, 2014, p.59).

Herman e Miyazaki (2007) descrevem as funções dos psicólogos no atendimento dos indivíduos com câncer. São elas:

- Favorecer a adaptação dos limites e das mudanças impostos pela doença e da adesão ao tratamento;

- Auxiliar no manejo da dor e do estresse associados à doença eaos procedimentos necessários; auxiliar na tomada de decisões;
- Preparar o paciente para a realização de procedimentos invasivos dolorosos e enfrentamento de possíveis consequências dos mesmos;

- Promover melhoria da qualidade de vida; auxiliar a aquisição de novas habilidades ou retomada de habilidades preexistentes; e revisão de valores para o retorno à vida profissional, familiar e social ou para o final da vida.

A atuação do psicólogo não se restringe ao paciente oncológico. É fundamental incluir a família no atendimento. Costa (2012) pontua, em relação à família do paciente oncológico, que a intervenção do psicólogo tem por finalidade oferecer um suporte emocional para que a mesma possa enfrentar de forma menos dolorosa possível à situação, pois comumente vivencia temores relacionados ao câncer e ao doente. Cada integrante do sistema familiar vivenciará e perceberá a situação do câncer de formas distintas, uma vez que o ser humano é dotado de subjetividade e que esta influencia diretamente sua maneira de compreender o mundo a sua volta. Segundo o autor, existem diversas formas para essa intervenção, sendo os atendimentos individuais e os grupos terapêuticos indicados individualmente, de acordo com a necessidade de cada familiar.

Nesta perspectiva, atuando junto ao sistema familiar, o psicólogo busca reforçar os vínculos afetivos entre família e paciente, facilitando um diálogo verdadeiro, capacitando-os a compartilhar experiências e emoções. Menezes et al. (2007) ressaltam que o oferecimento de um sistema de suporte à família, que facilite a compreensão do processo da doença em todas as fases, visa diminuir o impacto que os sintomas psicológicos causam no doente. Desta maneira, ainda segundos os autores, uma comunicação eficiente com a equipe de saúde e o máximo de informações sobre o tratamento e os cuidados específicos ao paciente, são aspectos inclusos nesse sistema de suporte à família oferecido pelo psicólogo, visto que a falta de informações sobre a condição do paciente é um dos fatores que causa maior estresse e desencadeia a ansiedade familiar.

Ainda sobre a atuação do Psico-oncologista, Peçanha (2005) ressalta que, além da atenção dada ao paciente e ao seu familiar, outro foco de intervenção é a equipe de saúde envolvida. Este trabalho envolve a permanente troca de informações a respeito do paciente e a colaboração mútua. Falhas na comunicação entre paciente, família e equipe podem prejudicar o estado emocional, principalmente, do paciente. O objetivo de sua atuação, portanto, é: “[...] minimizar o risco de disfuncionalidade no sistema pessoal e familiar pela ressignificação e pela elaboração da situação, favorecendo assim a qualidade de vida das pessoas envolvidas no processo". (PEÇANHA, 2005, p. 214).

Neste sentido, é relevante a interação entre todos os profissionais da equipe envolvidos no tratamento do câncer, garantindo assim um resultadopositivo, no 
que diz respeito a todos os aspectos implicados no adoecimento. Moraes (1994) intensifica a atribuição do psicólogo no cuidado oncológico junto à equipe multidisciplinar:

O papel do psicólogo é o de auxiliar a equipe na tomada de consciência das várias situações que acontecem na instituição hospitalar, no trocar de experiências, no debater as questões teóricas, filosóficas, morais, éticas, religiosas e, principalmente, no discutir diferentes abordagens, buscando uma compreensão psicodinâmica da comunicação na equipe (MORAES, 1994, p.60).

O vivenciar de um câncer, "revela a relação com o intraduzível da proximidade da morte, do inimigo cujo potencial destrutivo é desconhecido" (INCA,2014, p.58). O psicólogo que atua nesse contexto deve focar no problema emocional provocado pela doença e assim considerar e compreender o sujeito como um todo, analisando seu sofrimento físico, psíquico, suas limitações, angústias e conflitos, sempre visando à reabilitação do paciente de acordo com sua nova condição de vida. Desta forma, a psico-oncologia está pautada em trabalhar com os questionamentos surgidos no decorrer da vivência de pacientes, familiares e profissionais envolvidos em qualquer um dos estágios de adoecimento do câncer, seja ele no diagnóstico, no tratamento ou na fase terminal.

\section{Conclusão}

Neste trabalho foi possível perceber as contribuições da psico-oncologia para as questões ora debatidas, sendo esta uma área de fundamental importância na manutenção do bem estar psicológico do paciente e seus familiares em todo o processo de adoecimento do câncer.

$\mathrm{O}$ vivenciar de um câncer poderá desestruturar e abalar psiquicamente a vida do doente e de seus familiares, podendo colocá-los em fragilidade pelo próprio diagnóstico da doença. A partir do levantamento da literatura revisada, vimos que a família do paciente oncológico desenvolve um papel de suporte psicossocial importante e é essencial para a adesão ao tratamento e o enfrentamento do câncer.

Desta forma, o psicólogo que atua na área oncológica insere-se como um profissional fundamental na assistência para a família, para o paciente e também para os profissionais envolvidos no percurso do tratamento. Como vimos, este profissional vem buscando novas formas de intervenção com o objetivo de informar, tratar e identificar fatores que possam propiciar bem-estar e uma melhoria na qualidade de vida dos envolvidos neste processo. É importante que o psicólogo tenha uma boa comunicação com o paciente, com os familiares e com a equipe multidisciplinar, a fim de facilitar a compreensão do processo da doença em todas as fases.

No decorrer deste estudo, verificamos que a atuação do psicólogo no cuidado oncológico é fundamentada em proporcionar apoio psicossocial e psicoterapêutico no processo de enfrentamento do câncer. Sua atuação está voltada para a escuta, o acolhimento eos grupos de apoio, entre outras práticas;desta forma poderá minimizar os efeitos causados pela doença e também poderá dar um novo significado ou (re)significar este momento, que muitas vezes pode ser de angustia. Por ser uma doença crônica e grave, pode causar desequilíbrio na vida dos sujeitos, tanto social quanto emocionalmente, e a ponte para que algumas questões sejam resolvidas se dá através deste profissional.

Neste contexto, cabe enfatizar que a prática da Psico-oncologia é uma área de fundamental importância. Cabe ao psicólogo enxergar e compreender o paciente além da patologia, afinal, como se pode pensar, ele é muito mais que um tumor. Suas questões e angústias, se houver, deverão ser escutadas e, através deste profissional, estes pacientes podem conseguir fazer o atravessamento da doença de uma forma melhor e com um significado que o próprio paciente dará a seu tratamento. Através da Psico-oncologia o paciente será assistido de forma mais humanizada e única, atribuindose a este cuidado o modelo biopsicossocial.

Por fim, espera-se que este trabalho desperte interesse e incentive a realização de novos estudos na área da Psico-oncologia. Portanto, relata-se a necessidade de futuras pesquisas que abordem em específico a atuação do psicólogo frente ao processo de adoecimento do câncer no contexto psicossocial.

\section{Referências}

BRASIL. Ministério da Saúde. Secretaria Nacional de Ações Básicas de Saúde. Conceitos e Definições em Saúde. Brasília. 1977.

CAMPOS, E. M. P. A Psico-Oncologia: Uma nova visão do câncer- uma trajetória. São Paulo: Universidade de São Paulo, 2010.

CFP. Conselho Federal de Psicologia. Resolução nº 013/2007. Institui a Consolidação das Resoluções relativas ao Título Profissional de Especialista em Psicologia e dispõe sobre normas e procedimentos para seu registro. Brasília, 2007.

COSTA, A. L. J. O desenvolvimento da psico-oncologia: implicações para a pesquisa e intervenção profissional em saúde. Psicol. cienc. prof., v. 21, n. 2, p. 36-43, 2001.

COSTA, F. F. L. Câncer Infantil: Sentimentos, Vivências e Saberes do Familiar/Cuidador. Universidade Católica Dom Bosco. Campo Grande/MS, 2012.

DIAS, M. R.; DURÀ, E. Territórios da Psicologia Oncológica. Lisboa: Climepsi, 2001.

FAQUINELLO, P.; HIGARASHI, I. H.; MARCON, S. S. O atendimento humanizado em unidade pediátrica: percepção do acompanhante da criança hospitalizada. Revista Texto \& contexto de enfermagem, Florianópolis, v. 16, n. 4, out./dez. 2007.

FROELICH, T. C. Psico-oncologia e terminalidade: casos em que o paciente é uma criança. Jornada de Pesquisa em Psicologia, UNISC. Santa Cruz do 
Sul, 2011.

GIMENES, M. G. Definição, foco de estudo e intervenção. Em: M. M. M. J. Carvalho (org.). Introdução à Psiconcologia. Campinas: Livro Pleno, 2003. p.35-36.

HERMAN, A. R. S; MIYAZAKI, M. C. O. S. Intervenção psicoeducacional em cuidador de criança com câncer: relato de caso. Revista Arquivos de Ciências da Saúde, v. 14, n. 4, p. 238-244, 2007.

INCA. Instituto Nacional de Câncer José Alencar Gomes da Silva. Hospital do Câncer I. Seção de Psicologia. Sofrimento psíquico do paciente oncológico: o que há de específico? Rio de Janeiro, 2014.

KOVÁCS, M. J. Morte e desenvolvimento humano. São Paulo: Casa do Psicólogo, 1992.

KUBLER-ROSS, E. Sobre a Morte e o Morrer. São Paulo: Martins Fontes, 2005.

LIBERATO, R. P.; CARVALHO, V. A. Terapias integradas à oncologia. In: CARVALHO, V. A. et al. (org.). Temas em psico-oncologia. São Paulo: Summus, 2008.

MELMAN, J. Família e doença mental: repensando a relação entre profissionais de saúde e familiares. São Paulo: Escrituras, 2001.

MENEZES, C. N. B.; PASSARELLI, P. M.; DRUDE, F. S.; SANTOS, M. A.; VALLE, E. R. M. Câncer infantil: Organização familiar e doença. Revista Mal-Estar e Subjetividade, v. 7, n. 1, p. 191-210, 2007.

MORAES, M. C. O paciente oncológico, o psicólogo e o hospital. In: CARVALHO, M. M. M. J. Introdução à Psiconcologia. São Paulo: Editorial PsyII, 1994.

NEME, C. M. B. Psico-oncologia: caminhos, resultados e desafios da prática. In: Psico-oncologia: caminhos e perspectivas. São Paulo: Summus, 2010.

PEÇANHA, D. L. N. Câncer: recursos de enfrentamento na trajetória da doença a difícil trajetória. In: CARVALHO, V. A. (org.). Temas em Psicooncologia. São Paulo: Summus, 2005.

SAMPAIO, A. S.; LOHR, S. S. Atuação em casas de apoio: pensando o papel da psicologia e construindo novos caminhos. Rev. RUBS., Curitiba, v. 1, n. 3, p. 52-60, set./Dez, 2008.

SAWADA, N. O.; NICOLUSSI, A. C.; OKINO, L. O.; CARDOSO, F. M. C.; ZAGO, M. M. F. Avaliação da Qualidade de Vida de Pacientes com Câncer submetidos a Quimioterapia. Rer. Esc. Enferm. USP, v. 43, n. 3, p. 581-587,2009.

SIMONETTI, A. Manual de Psicologia Hospitalar: o mapa da doença. São Paulo: Casa do Psicólogo, 2004

VEIT, M. T.; CARVALHO, V. A. Psico-Oncologia: um novo olhar para o câncer. O Mundo da Saúde, São Paulo, 2010. Disponível em: http://www. saocamilo-sp.br/pdf/mundo saude/79/526a530.pdf. Acesso em: $22 \mathrm{fev}$. 2019. 